\title{
Is preinfarction angina related to the presence or absence of coronary plaque rupture?
}

\author{
S Kojima, H Nonogi, Y Miyao, S Miyazaki, Y Goto, A Itoh, S Daikoku, T Matsumoto, \\ I Morii, C Yutani
}

Objective-To analyse the prodrome of acute myocardial infarction in relation to the plaque morphology underlying the infarct.

Design-A retrospective investigation of the relation between rupture and erosion of coronary atheromatous plaques and the clinical characteristics of acute myocardial infarction. The coronary arteries of 100 patients who died from acute myocardial infarction were cut transversely at $3 \mathrm{~mm}$ intervals. Segments with a stenosis were examined microscopically at $5 \mu \mathrm{m}$ intervals. The clinical features of the infarction were obtained from the medical records.

Results-A deep intimal rupture was encountered in 81 plaques, whereas 19 had superficial erosions only. There were no differences in the location of infarction, the incidence of hypertension, diabetes mellitus, or hyperlipidaemia, diameter stenosis of the infarcted related artery, Killip class, Forrester's haemodynamic subset, or peak creatine kinase between plaque rupture and plaque erosion groups. The presence of plaque rupture was associated with significantly greater incidences of leucocytosis, current smoking, and sudden or unstable onset of acute coronary syndrome. In patients with unstable preinfarction angina, new onset rest angina rather than worsening angina tended to develop more often in the plaque rupture group than in the plaque erosion group $(\mathrm{p}=0.08)$.

Conclusions-Plaque rupture causes the sudden onset of acute myocardial infarction or unstable preinfarction angina, which may be aggravated by smoking and inflammation.

(Heart 2000;83:64-68)

Keywords: acute coronary syndrome; plaque rupture; white blood cell; preinfarction angina

Changes in plaque morphology consisting of plaque rupture or fissure have been recognised as important mechanisms of coronary thrombosis in acute myocardial infarction, unstable angina pectoris, and cardiac sudden death. ${ }^{1-8}$ Vulnerable plaque has been reported to consist of a thin fibrous cap and lipid rich core, ${ }^{35-9}$ and plaque rupture of the thin fibrous cap is believed to result in coronary thrombosis. However, coronary plaque erosion (superficial intimal injury) without coronary rupture has been reported as a cause of coronary artery thrombosis, ${ }^{10-12}$ and the immediate site of plaque rupture or erosion is always marked by an inflammatory process. ${ }^{11} 12$

The relation between the morphology of plaque disruption and clinical presentation of acute coronary syndrome has not been examined in detail. If vulnerable plaque is formed and its rupture and subsequent thrombosis are triggered by some specific factors, there should be corresponding characteristic clinical features in patients with plaque rupture. Therefore, we investigated whether patients with acute myocardial infarction on the basis of plaque rupture have different prodromal signs and symptoms leading up to the acute infarct from those with plaque erosions.

\section{Methods}

\section{PATIENTS}

The study group consisted of 100 patients (74 men, 26 women, mean (SD) age 69 (10) years) who had died of acute myocardial infarction between 1978 and 1995, within one month of the onset of the infarct. Necropsies on all these patients were performed at the National
Cardiovascular Centre. Acute myocardial infarction was defined as typical chest symptoms lasting more than 30 minutes, accompanied by ST-T wave changes or abnormal $Q$ waves on the ECG and by a rise in creatine kinase to more than twice the upper normal limit. None of the patients had received any interventional treatment. The median survival time from the onset was 98 hours.

EVALUATION OF THE CORONARY ARTERIES All the hearts were studied by the pathologists at the National Cardiovascular Centre. They were examined fresh and uncut, with fixation performed on the time of necropsy. The coronary arterial tree was perfusion fixed with $10 \%$ neutral buffered formalin overnight at $100 \mathrm{~mm}$ Hg. ${ }^{6717}$ Postmortem angiography with barium gelatin was performed in all cases. The major epicardial coronary arteries (left main, left anterior descending, left circumflex, and right coronary arteries) and their major branches (left diagonal branches, left obtuse marginal branches, and posterior descending coronary artery) were cut transversely at $3 \mathrm{~mm}$ intervals macroscopically (following decalcification if necessary). The segments that had a stenosis were submitted to light microscopy, histological examination, and morphometric measurements and were cut at $5 \mu \mathrm{m}$ intervals, and stained with haematoxylin-eosin.

\section{DEFINITIONS}

The culprit lesion was defined both clinically and pathologically as the lesion that had acute thrombus formation on the ruptured coronary plaque or the plaque erosion. When the
Accepted for publication 21 July 1999 
electrocardiography, echocardiography, and/or coronary angiography had been examined on admission after the onset of acute myocardial infarction, they were used to diagnose acute myocardial infarction and to determine the culprit lesion (coronary angiography had been examined in 80 patients during the acute phase).

Plaque morphology was classified into two categories based on the pathological examination: coronary plaque rupture and plaque erosion. Coronary plaque rupture was defined as a disruption of a fibrous cap on a lipid core. ${ }^{67}$ Figure 1A shows a typical example of acute coronary plaque rupture. The lumen is separated from the underlying lipid core by a thin fibrous cap. The rupture site, which was characterised by discontinuity of the fibrous cap, was observed at the shoulder lesion of the plaque and was in contact with a lumen occupying thrombus on the lipid-rich core. At higher magnification the connection between the platelet-rich thrombus and the lipid core was through a disrupted thin fibrous cap infiltrated by macrophages. Plaque erosion was defined as a superficial erosion in direct contact with acute thrombus without rupture of a lipid pool. ${ }^{67}$ Figure $1 \mathrm{~B}$ shows a typical example of plaque erosion. The hard plaque which includes some parts of calcification is recognised. A lipid core was present deep within the plaque but there was no communication between the thrombus and lipid core. At higher magnification the luminal surface was focally eroded and a part of the endothelium was lost and the thrombus which predominantly consists of platelets was in contact with eroded plaque.

The arterial size (defined by the area within the internal elastic lamina) and the lumen area (excluding the thrombus) were measured and determined by the percent stenosis of the cross sectional area [100(1-lumen area/arterial size)] in the culprit lesion.

A

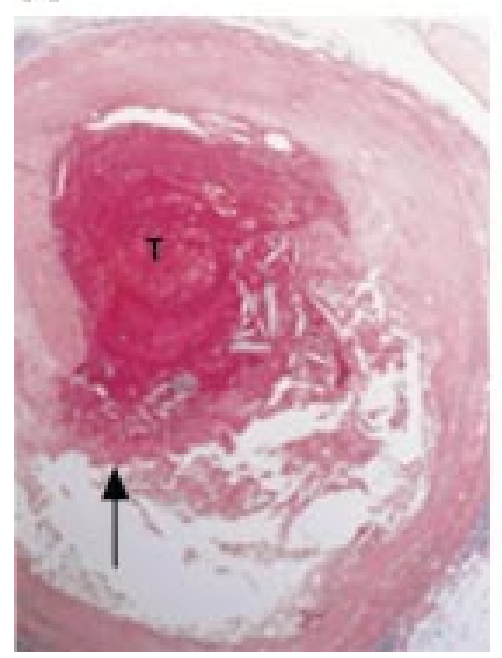

B

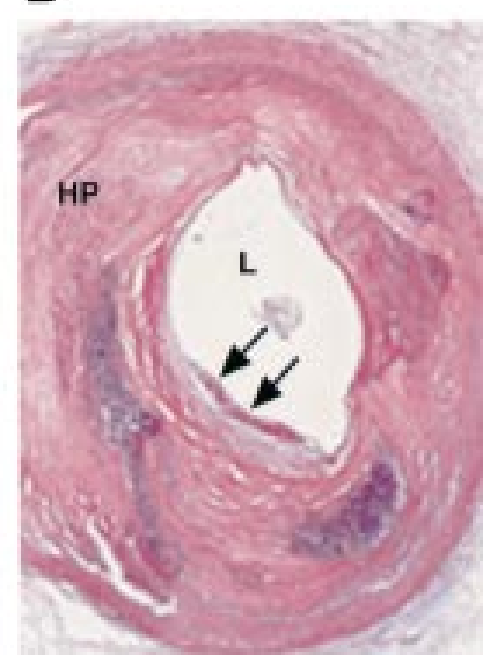

Figure 1 (A) A coronary plaque rupture was found in a patient who died one week after the onset of acute myocardial infarction. At lower magnification the rupture site (arrow) is seen at the shoulder lesion, which is considered to have had a thin fibrous cap. An occlusive thrombus (T) was present (haematoxylin and eosin stain). (B) A coronary plaque erosion was found in a patient who died two weeks after the onset of acute myocardial infarction. At lower magnification, a concentric hard plaque (HP) which includes focal calcification was present. The luminal $(L)$ plaque surface was in contact with non-occlusive thrombus (arrows) (haematoxylin and eosin stain).
CLINICAL CHARACTERISTICS

Clinical characteristics and laboratory findings were assessed from medical records of each patient and compared between the plaque rupture group and the plaque erosion group. The status of preinfarction angina was classified into three categories. Sudden onset was defined as an onset of acute myocardial infarction without preinfarction angina. Unstable angina was defined as worsening angina or new onset of angina within the preceding four weeks. Stable angina was defined as angina not included in the above categories. As the presence of stable angina suggests the existence of an organic stenosis in at least one coronary artery, and as sudden onset and unstable angina are suggestive of coronary plaque rupture, patients who were categorised as "sudden onset" and "unstable angina" were combined in the subsequent analysis. The presence or absence of risk factors for coronary artery disease was determined as follows. Hypertension was considered to be present when long term treatment had been given or was required. Diabetes mellitus was defined by a previous physician's diagnosis and treatment with insulin, oral hypoglycaemic agents, or diet. Hypercholesterolaemia was defined as a serum total cholesterol of at least $5.7 \mathrm{mmol} / 1$. History of smoking was defined as a current smoker. Other clinical and laboratory findings included previous infarction and peak creatine kinase, the number of diseased coronary arteries, Killip's class and Forrester's haemodynamic subset, and white blood cell count on admission.

\section{STATISTICAL ANALYSIS}

Numerical data are presented as mean (SD) unless otherwise stated. Continuous variables and categorical variables were compared with Student's $t$ test and the $\chi^{2}$ test, respectively. A p value $\leqslant 0.05$ was considered significant. For multivariate analysis, the effects of risk factors such as age, sex, the presence or absence of hypertension, diabetes mellitus, and cigarette smoking, total cholesterol, and the status of preinfarction angina on plaque morphology were analysed in all 100 patients using multiple logistic regression analysis.

\section{Results}

There were 81 cases with coronary plaque rupture and 19 with plaque erosion. Age and the incidence of hypertension, diabetes mellitus, and hyperlipidaemia (total cholesterol) were not different between patients with plaque rupture and those with plaque erosion. The incidence of male patients was higher in the plaque rupture group than in the plaque erosion group, but the difference was not statistically significant $(78 \% v$ $58 \%, \mathrm{p}=0.08)$. The coronary plaque rupture group had a higher rate of active smokers $(62 \%)$ than the plaque erosion group $(26 \%, \mathrm{p}=0.005)$ (table 1).

The incidence of previous myocardial infarction, Killip's class, Forrester's haemodynamic subset, the number of diseased coronary arteries, percentage stenosis, peak creatine kinase, and the site of the culprit lesion were not 
Table 1 Risk factors, clinical background, and type of coronary thrombosis in 100 patients with acute myocardial infarction

\begin{tabular}{llll}
\hline & $\begin{array}{l}\text { Plaque rupture } \\
(n=81)\end{array}$ & $\begin{array}{l}\text { Plaque erosion } \\
(n=19)\end{array}$ & $p$ Value \\
\hline Age (years) & $68.6(9.6)^{\star}$ & $71.2(10.0)^{\star}$ & NS \\
Sex (male/female) & $63 / 18$ & $11 / 8$ & 0.08 \\
Hypertension (\%) & 56 & 58 & $\mathrm{NS}$ \\
Diabetes mellitus (\%) & 61 & 42 & $\mathrm{NS}$ \\
Hyperlipidaemia (\%) & 46 & 50 & $\mathrm{NS}$ \\
Total cholesterol (mmol/1) & $5.52(1.35)^{\star}$ & $5.10(1.09)^{\star}$ & $\mathrm{NS}$ \\
Cigarette smoking (\%) & 62 & 26 & 0.005 \\
Forrester's haemodynamic subset (I/II/III/IV) & $17 / 8 / 10 / 27$ & $4 / 2 / 4 / 5$ & $\mathrm{NS}$ \\
Killip's class (I/II/III/IV) & $22 / 17 / 9 / 33$ & $4 / 3 / 3 / 9$ & $\mathrm{NS}$ \\
Multivessel disease (\%) & 76 & 92 & $\mathrm{NS}$ \\
Stenosis (\%) & $76(16)^{\star}$ & $70(19)^{\star}$ & $\mathrm{NS}$ \\
Peak creatine kinase (U/1) & $4693(3492)^{\star}$ & $3310(1687)^{\star}$ & $\mathrm{NS}$ \\
Previous myocardial infarction (\%) & 49 & 42 & $\mathrm{NS}$ \\
\hline
\end{tabular}

${ }^{\star}$ Mean (SD).

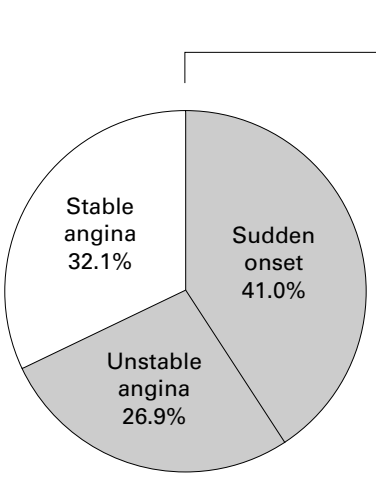

Plaque rupture group $(\mathrm{n}=78)$ $p=0.02$

Figure 2 Pie charts showing the status of preinfarction angina. The patients with plaque rupture had a significantly higher incidence of sudden onset and unstable angina than those with plaque erosion.
Plaque rupture group $(n=21)$

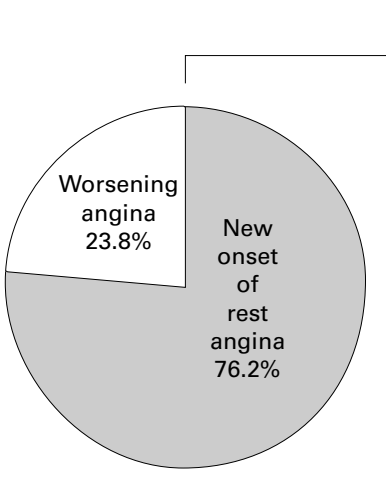

$p=0.08$

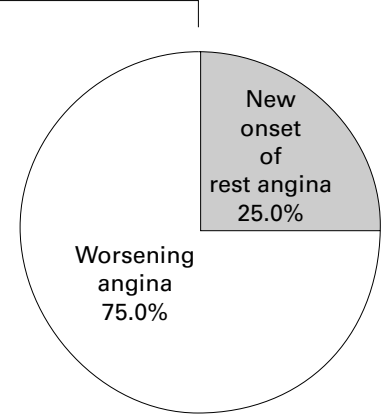

Plaque erosion group

$(n=4)$
Figure 3 Pie charts showing the status of unstable angina. The patients with plaque rupture had a higher incidence of new onset of rest angina than those with plaque erosion, but the difference was not significant.

Table 2 Risk factors, status of preinfarction angina, and the type of coronary thrombosis in 100 patients with acute myocardial infarction using univariate and multivariate analyses

\begin{tabular}{lllll}
\hline & $\begin{array}{l}\text { Plaque rupture } \\
(n=81)\end{array}$ & $\begin{array}{l}\text { Plaque erosion } \\
(n=19)\end{array}$ & $\begin{array}{l}\text { Univariate } \\
\text { analysis }(p)\end{array}$ & $\begin{array}{l}\text { Multivariate } \\
\text { analysis }(p)\end{array}$ \\
\hline Age (years) & $68.6(9.6)^{\star}$ & $71.2(10.0)^{\star}$ & 0.31 & 0.61 \\
Sex (male/female) & $63 / 18$ & $11 / 8$ & 0.08 & 0.39 \\
Hypertension (\%) & 56 & 58 & 0.90 & 0.62 \\
Diabetes mellitus (\%) & 61 & 42 & 0.14 & 0.95 \\
Total cholesterol (mmol/l) & $5.52(1.35)^{\star}$ & $5.10(1.09)^{\star}$ & 0.25 & 0.64 \\
Cigarette smoking (\%) & 62 & 26 & 0.005 & 0.04 \\
Preinfarction angina (\%) & 68 & 38 & 0.02 & 0.02 \\
\hline
\end{tabular}

${ }^{\star}$ Mean (SD). significantly different between the two groups (table 1). The mean (SD) survival time from the onset of acute myocardial infarction was not different between the two groups (186 (260) hours in the patients with plaque rupture, 159 (184) hours in the patients with plaque erosion). More than $50 \%$ of the causes of death were cardiogenic shock, followed by cardiac rupture and arrhythmia in both groups, and the distribution of causes was not significantly different between the two groups.

STATUS OF PREINFARCTION AND TYPE OF CORONARY PLAQUE DISRUPTION

Among the 100 patients enrolled in the study, there were six patients (three in the plaque rupture group and three in the plaque erosion group) without detailed descriptions about preinfarction angina. The patients with plaque rupture had a higher incidence of sudden onset of infarction than those with plaque erosion $(41 \%$ v $13 \%, \mathrm{p}=0.04)$. On the other hand, the patients with plaque erosion had a higher incidence of stable angina than those with plaque rupture $(63 \%$ v $32 \%, \mathrm{p}=0.02)$. Among patients with sudden onset or unstable angina, those with plaque rupture did not have a significantly higher incidence of sudden onset than those with plaque erosion (fig 2). In 25 patients with unstable preinfarction angina, the patients with plaque rupture had a higher incidence of new onset of rest angina than those with plaque erosion, but the difference was not significant $(76 \% v 25 \%, \mathrm{p}=0.08$ ) (fig 3).

By multivariate analysis, being an active smoker (odds ratio, 5.3) and having preinfarction angina (odds ratio, 4.8) were independently associated with plaque rupture (table 2).

Data on the association between white blood cell count and plaque morphology were available in 65 patients within six hours of the onset of acute myocardial infarction. The mean count of white blood cells was significantly higher in patients with plaque rupture than in those with plaque erosion (12 423 (4502) $v 9093$ (3162) cells $/ \mathrm{mm}^{3}, \mathrm{p}=0.02$ ) (fig 4). The average time from the onset of acute myocardial infarction to admission was not significantly different between the two groups (3.1 (1.4) v 3.0 (1.2) hours).

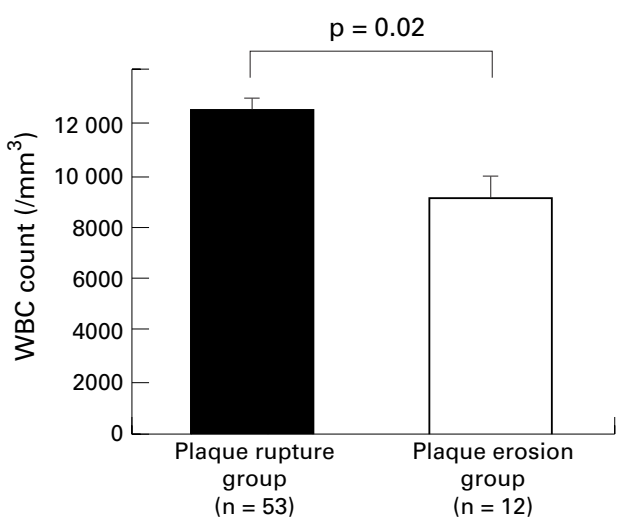

Figure 4 White blood cell count within six hours of the onset of acute myocardial infarction. Error bars $=$ SEM. 


\section{Discussion}

In this study, we found coronary plaque rupture in $81 \%$ of cases of death after acute myocardial infarction. Sudden onset of acute myocardial infarction or unstable preinfarction angina were more often seen in the plaque rupture group than in the plaque erosion group. The plaque rupture group contained a significantly higher number of active smokers and a higher white blood cell count on admission than the plaque erosion group.

ACUTE MYOCARDIAL INFARCTION AND PLAQUE DISRUPTION

Vulnerable plaque is characterised by increased numbers of macrophages which release matrix metalloproteinases such as interstitial collagenase, gelatinase, and stromelysin, increased expression of the tissue factor, reduced numbers of smooth muscle cells, a lipid core that occupies a high proportion of the overall plaque volume, and a thin plaque cap. ${ }^{12-15}$ Thus rupture of a vulnerable plaque and the following thrombus formation are thought to be the most important mechanisms underlying the sudden onset of acute coronary syndrome. In acute coronary death with coronary thrombus, Davies has reported that plaque rupture was present in $75 \%$ of cases and the presence of superficial intimal injury was observed in the remaining $25 \%$. $^{10}$ Another pathological study has indicated that plaque rupture was present in $78 \%$ of cases and the absence of plaque rupture was observed in the remaining $22 \%{ }^{16}$ These rates are similar to the findings of the present study, but contrast markedly with previous work by Farb et al, who showed that about $50 \%$ were caused by plaque erosion. ${ }^{17}$ Intriguingly, Farb found that in women who died suddenly of ischaemic heart disease ( $n=16(32 \%)$ of 50 cases), $69 \%$ of the thrombi were associated with plaque erosion. Burke et al studied only men with coronary thrombus who had died suddenly, from myocardial ischaemia and showed that $31 \%$ of cases were caused by plaque erosion. ${ }^{7}$ In contrast, Burke and colleagues recently reported only women with acute coronary thrombus who had died suddenly, and showed that $69 \%$ of these cases were caused by plaque erosion. ${ }^{18}$ In their study, $83 \%$ of cases of plaque erosion were in young or premenopausal women (less than 50 years of age). In the present study, 26 cases were in women who were more than 60 years of age and postmenopausal, and plaque erosion had a tendency to be seen more often in women $(31 \%$ in women and $15 \%$ in men, $p=0.08)$. Thus these findings suggest that sex influences the differences in plaque morphology in acute coronary syndrome.

PREINFARCTION STATE AND PLAQUE

MORPHOLOGY

In the present study, the plaque rupture group had a higher incidence of sudden onset acute myocardial infarction or unstable preinfarction angina. In a study by Capone et al, the incidence of thrombus by angiographic analysis was higher in patients with a short duration of unstable angina or with recent onset of rest angina than in those with slowly progressive preinfarction angina or a period of stable angina. ${ }^{19}$ In addition, Falk et al reported that patients who died with unstable angina had layered thrombus with plaque disruption. ${ }^{5}$ However, there has been no study on the relation between preinfarction angina and plaque morphology in acute myocardial infarction. Thus the present results suggest that patients with unstable preinfarction angina or sudden onset acute myocardial infarction are likely to have more fresh thrombus overriding plaque disruption than those with stable or slowly progressive preinfarction angina.

\section{SMOKING, WHITE BLOOD CELL COUNT, AND}

PLAQUE MORPHOLOGY

Burke et al reported that smoking was an independent risk factor for thrombosis irrespective of plaque morphology. ${ }^{7}$ However, their analysis was limited to men, who showed a high incidence of smoking. In the present study of both sexes, the plaque rupture group contained a significantly larger number of cigarette smokers than the plaque erosion group. In men, the incidence of coronary plaque rupture was slightly higher in cigarette smokers than in non-smokers ( $89 \%$ v $78 \%$ ), but the difference was not statistically significant. In contrast, in women the incidence of coronary plaque rupture was significantly higher in cigarette smokers than in non-smokers (100\% v 56\%, $\mathrm{p}=0.03)$. Thus it is suggested that in women cigarette smoking may be linked with coronary plaque rupture and acute thrombosis.

In the present study, the white blood cell count on admission within six hours of the onset of acute myocardial infarction was higher in the plaque rupture group than in the plaque erosion group. Leucocytes are the major mediators of inflammation. Adhesion of circulating leucocytes to vascular endothelium is considered to be a key step in endothelial injury. Furthermore, the activity of constitutional cell components at the site of plaque rupture has been reported to reflect the inflammatory intensity or the interactions among the components at the site of gruel plaque. ${ }^{62-1520}$ Neutrophils secrete proteolytic enzymes that can destroy tissue, ${ }^{12}$ but they are seldom encountered in the intact plaque. ${ }^{21}$ Neutrophils probably enter the plaque tissue shortly after plaque disruption. Recently, Liuzzo et al and Haverkate et al reported that the modest acute phase responses of $\mathrm{C}$ reactive protein are probably not the result of myocardial necrosis, and that $\mathrm{C}$ reactive protein values in unstable angina may reflect the inflammatory activity within the coronary arteries. ${ }^{22} 23$ Thus a rise in the white blood cell count during the very early phase of acute myocardial infarction may reflect either inflammation in the vulnerable plaque or rupture of the plaque.

\section{LIMITATIONS OF THE STUDY}

Potential limitations are as follows. First, not all the patients died suddenly (that is, within 24 hours of the onset of acute myocardial infarction). This might be responsible for the 
different plaque morphologies, in addition to the fact that there were more young women in previous studies. ${ }^{17}{ }^{18}$ In this study, 30 patients died within 24 hours of the onset of acute myocardial infarction, $24(30 \%)$ in the plaque rupture group and six $(32 \%)$ in the plaque erosion group. The incidence of sudden onset or unstable preinfarction angina and of cigarette smoking, in which there were significant differences between the plaque rupture group and the plaque erosion group by univariate and multivariate analyses, were not significantly different between patients dying within 24 hours of the onset of symptoms (group A) and those dying after more than 24 hours (group B) (64\% $v 62 \%$, and $57 \%$ v 54\%, respectively). Furthermore, fresh occluding thrombi were found more often in patients dying during the acute stage of infarction, whereas organised thrombi were found in patients surviving more than one month. ${ }^{24}{ }^{25}$ Therefore plaque morphology is unlikely to be modified within a month of the onset of acute myocardial infarction, so group A and group B can be analysed statistically as one group. Second, the white blood cell count may reflect not only inflammation in the vulnerable plaque or plaque rupture itself but also the developing myocardial necrosis at the infarcted area or myocardial damage.

CONCLUSIONS

It has been shown that patients with acute myocardial infarction associated with coronary plaque rupture are more likely to have sudden onset or unstable angina, to be active smokers, and to have a higher white blood cell count within six hours of onset than patients with plaque erosion. These findings suggest that plaque rupture might cause the sudden onset of acute myocardial infarction or unstable preinfarction angina which may be aggravated by smoking and inflammation.

1 Shah PK, Forrester JS. Pathophysiology of acute coronary syndromes. Am f Cardiol 1991;68:16-23C.

2 Report of a meeting of physicians and scientists, Texas Heart Institute and the University of Texas Medical School at Houston. Thrombus and unstable angina. Lancet 1993; 342:1151-5.

3 Fuster V, Lewis A. Conner Memorial Lecture. Mechanisms Fuster V, Lewis A. Conner Memorial Lecture. Mechanisms leading to myocardial infarction: insights from

4 Muller JE, Abela GS, Nesto RW, et al. Triggers, acute risk factors and vulnerable plaques: the lexicon of a new frontier. $\mathcal{F}$ Am Coll Cardiol 1994;23:809-13.
5 Falk E, Shah PK, Fuster V. Coronary plaque disruption. Circulation 1995;92:657-71.

6 Davies MJ, Thomas A. Thrombosis and acute coronaryartery lesions in sudden cardiac ischemic death. $N$ Engl $f$ Med 1984;310:1137-40

7 Burke AP, Farb A, Malcom GT, et al. Coronary risk factors and plaque morphology in men with coronary disease who died suddenly. N Engl f Med 1997;336:1276-82.

8 Kristensen SD, Ravn HB, Falk E. Insights into the pathophysiology of unstable coronary artery disease. $A m \mathcal{F}$ Cardiol 1997;80(5A):5-9E.

9 Gertz SD, Roberts WC. Hemodynamic shear force in rupture of coronary arterial atherosclerotic plaques. $A m \mathcal{F}$ Cardiol 1990;66:1368-72.

10 Davies MJ. A macro and micro view of coronary vascular insult in ischemic heart disease. Circulation 1990;82(suppl II):II-38-46.

11 Davies MJ. Thrombosis and coronary atherosclerosis. In: Julian D, Kubler W, Norris RM, et al, eds. Thrombolysis in cardiovascular disease. New York: Marcel Dekker, 1989: $25-43$

12 van der Wal AC, Becker AE, van der Loos CM, et al. Site of intimal rupture or erosion of thrombosed coronary atherosclerotic plaques is characterized by an inflammatory process irrespective of the dominant plaque morphology. Circulation 1994;89:36-44.

13 Galis ZS, Sukhova GK, Lark MW, et al. Increased expression of matrix metalloproteinases and matrix degrading activity in vulnerable regions of human atherosclerotic plaques. F Clin Invest 1994;94:2493-503.

14 Moreno PR, Bernardi VH, López-Cuéllar J, et al. Macrophages, smooth muscle cells, and tissue factor in unstable angina: implications for cell-mediated thrombogenicity in acute coronary syndromes. Circulation 1996;94:3090-7.

15 Annex BH, Denning SM, Channon KM, et al. Differential expression of tissue factor protein in directional atherectomy specimens from patients with stable and unstable coronary syndromes. Circulation 1995;91:619-22.

16 Falk E. Plaque rupture with severe pre-existing stenosis precipitating coronary thrombosis: characteristics of coronary atherosclerotic plaques underlying fatal occlusive thrombi. Br Heart f 1983;50:127-34.

17 Farb A, Burke AP, Tang AL, et al. Coronary plaque erosion without rupture into a lipid core: a frequent cause of coronary thrombosis in sudden coronary death. Circulation 1996;93:1354-63.

18 Burke AP, Farb A, Malcom GT, et al. Effect of risk factors on the mechanism of acute thrombosis and sudden coronary death in women. Circulation 1998;97:2110-16.

19 Capone G, Wolf NM, Meyer B, et al. Frequency of intracoronary filling defects by angiography in angina pectoris at rest. Am 7 Cardiol 1985;56:403-6.

20 Kovanen PT, Kaartinen M, Pavonen T. Infiltrates of activated mast cells at the site of coronary atheromatous rosion or rupture in myocardial infarction. Circulation 1995;92:1084-8.

21 Weiss SJ. Tissue destruction by neutrophils. $N$ Engl f Med 989;320:365-76

22 Liuzzo G, Biasucci LM, Rebuzzi AG, et al. Plasma protein acute-phase response in unstable angina is not induced by ischemic injury. Circulation 1996:94:2373-80.

23 Haverkate F, Thompson SG, Pyke SDM, et al, for the European Concerted Action on Thrombosis and Disabilities Angina Pectoris Study Group. Production of C-reactive protein and risk of coronary events in stable and unstable angina. Lancet 1997;349:462-6.

24 Horie T, Sekiguchi M, Hirosawa K. Coronary thrombosis in pathogenesis of acute myocardial infarction: histopathoogical study of coronary arteries in 108 necropsied cases using serial section. Br Heart f 1978;40:153-61.

25 Yutani C, Ishibashi-Ueda H, Konishi M, et al. Histopathological study of acute myocardial infarction and pathoetiology of coronary thrombosis: a comparative study in four districts in Japan. Fpn Circ $\mathcal{F}$ 1987;51:352-61. 